\title{
Contemporary therapy of atrial fibrillation
}

\author{
Xing Sheng Yang", Jing Ping Sun", Cheuk Man Yu ${ }^{* \#}$ \\ Division of Cardiology, Prince of Wales Hospital, The Chinese University of Hong Kong, Hong Kong, China \\ Email: ${ }^{\text {cmyu@ } @ \text { cuhk.edu.hk }}$
}

Received 23 April 2012; revised 28 May 2012; accepted 13 June 2012

\begin{abstract}
Atrial fibrillation (AF) is estimated that by 2010, approximately 2.6 million people will be affected in USA; by 2050, that number may increase to 10 million patients. Generally, rate control alone is reasonable in some AF patients, especially asymptomatic patients. Restoration and maintenance of sinus rhythm (SR) may be achieved by means of cardioversion, drugs or/ and catheter ablation. Pharmacological therapy can be useful to maintain SR and prevent tachycardiainduced cardiomyopathy. All patients with AF regardless of whether a rhythm or rate control strategy recommend anticoagulant, antiplatelet or both combined therapy for prevention of thromboembolism, except those with lone AF or contraindications. Drug selection should be based upon the absolute risk of stroke, bleeding, the relative risk and benefit for a given patient. Biventricular pacing may overcome many of the adverse hemodynamic effects associated with RV pacing alone. A target individual ectopic foci ablation within the pulmonary vein (PV) has evolved to circumferential electrical isolation of the entire PV musculature. Cavotricuspid isthmus should be considered as first-line therapy for patients with typical atrial flutter. Completely non-fluoroscopic ablation guided by Real-Time Magnetic Resonance Imaging (RTMRI) using a steerable and non-ferromagnetic catheter is a promising novel technology in interventional electrophysiology.
\end{abstract}

Keywords: Atrial Fibrillation; Antiarrhythmic Drug; Anticoagulant Therapy; Antiplatelet Therapy;

Cardioversion; Catheter Ablation

\section{INTRODUCTION}

Atrial fibrillation (AF) is the most common clinically significant cardiac rhythm disorder, and a progressive disease with higher mortality consequences. The clinical impact of the disease burden of AF includes hemody-

\footnotetext{
*Author contributions: Yang XS wrote the manuscript; Sun JP and Yu $\mathrm{CM}$ revised the manuscript.

${ }^{\#}$ Corresponding author.
}

namic and myocardial consequences, such as reduced cardiac output, and heart failure (HF), increased hospitalizations, reduced quality of life, increased risk of thromboembolism and decreased survival [1]. The frequency of AF is estimated that by 2010, approximately 2.6 million people will be affected in the United States [2], AF accounts for approximately one third of the hospitalizations related primarily to cardiac arrhythmias $[3,4]$. The prevalence of $\mathrm{AF}$ is expected to increase dramatically, by 2050, that number may increase to 10 million patients [2], reflecting the aging US population and rising prevalence of AF risk factors [5,6]. Cross-sectional studies have demonstrated an $8 \%$ prevalence among patients older than 80 years [7]. In addition to age, Framingham Study demonstrated that men had a 1.5 -fold greater risk of developing $\mathrm{AF}$ than women, and hypertension and diabetes were significant independent predictors of AF [8]. Despite the severe burden of disease and the potential for progression to a permanent state of arrhythmia, AF remains a highly treatable disorder, especially with early intervention [1].

The clinical type of AF can be classified as follows: Paroxysmal ( $\leq 7$ days) (most less than $24 \mathrm{~h}$ ), which is self-terminating; persistent ( $>7$ days), which requires intervention for termination; permanent, which is refractory to cardioversion (CV) or is accepted as the final rhythm or both paroxysmal and persistent AF may be recurrent [9]. Cardiac testing is required before initiation of therapy to identify potential causes and to help determine which drugs can be used safety during treatment. The potential causes include structural heart disease, coronary heart disease (CHD), ventricular preexcitation, Ion channel defects (e.g. such as Brugoda syndrome), and hyperthyroidism. The induced factors include conditions such as alcohol consumption and carbon monoxide poisoning etc.

The minimal initial evaluation should include the following tests: Electrocardiogram (ECG), transthoracic echocardiogram (TTE), blood tests, and additional testing that may be necessary in selected patients with $\mathrm{AF}$ includes: six-minutes walk test, exercise testing, Holter monitoring or event recording, transesophageal echocardiography (TEE), electrophysiological (EP) study and 
chest radiograph.

\section{MANAGEMENT OF PATIENTS WITH AF}

Management of patients with AF have become available, including recommendations for: strict versus lenient heart rate control, combined use of antiplatelet and anticoagulant therapy for prevention of thromboembolism. Maintenance of sinus rhythm (SR) can confer significant clinical benefits across a wide spectrum of AF patients. It has been associated with improved cardiac function, decreased hospitalization, and improvement in quality of life. If the patient can not maintain SR, it may be directly corrected with the structural atrial remodeling that occurs as a consequence of $\mathrm{AF}$ and, in turn, induces other electrophysiologic changes, then further AF occurs.

There are three kinds of remodeling in AF [10]: electrical, contractile and structural. Electrical remodeling occurs quickly and is often reversible. Structural remodeling can take several weeks, months, or even years to occur, that includes cellular changes such as fibrosis, myolysis, accumulation of glycogen, and cell enlargement and death. At a certain points in time, some of these changes are irreversible [11]. If the patients stay in $\mathrm{AF}$ for more than one year, recurrent $\mathrm{AF}$ after cardioversion (CV) frequently occurred, the reason may partly be some permanent damage that may be irreversible developed, or damage that is reversible can only be reversed if SR is maintained for longer periods of time, which may be $\mathrm{CV}$ with multiple times. Patients converted to SR within 3 months have a $69 \%$ chance of remodeling in 6 months compared to only $27 \%$ if patients are allowed to remain in AF for $>12$ months [12].

A rate control strategy alone is reasonable in some patients with $\mathrm{AF}$, especially those who are asymptomatic, but before making the decision, the doctor should consider how permanent $\mathrm{AF}$ is likely to affect the patients in the future [13]. A rate control allowing adequate time for ventricular filling and avoiding rate-related ischemia, may result in improved hemodynamics. A heart rate recording during submaximal or maximal exercise or 24hour Holter recording may be an option.

Antithrombotic therapy to prevent thromboembolism is recommended for all patients with AF regardless of whether a rhythm or a rate control strategy is chosen, except those with lone AF or contraindication. Selection of the antithrombolic agent should be based on the absolute risks of stroke, bleeding, relative risk and benefits for a given patients $[9,13]$. Combining anticoagulant with antiplatelet therapy are recently recommended [13].

\section{RESTORATION OF SINUS RHYTHM}

Principles of cardioversion (CV) are as followed: CV may be achieved by means of a drug or an electrical shock. Direct current CV is more effective than pharmacological CV. The more recent the onset of AF, the more effective is pharmacological CV. The primary disadvantage of electrical CV is that it requires sedation or anesthesia. The primary disadvantage of pharmacological CV is the risk of torsades de pointers. The risk of thromboembolism or stroke does not differ between pharmacological CV and electrical CV. Be prepared of significant sinus bradycardia after $\mathrm{CV}$ in patients on high dose AV nodal blocking drugs $[9,13]$.

Direct current CV should be used by shocks will be delivered synchronous to R-wave, and use of a biphasic defibrillator. The AF patients with ongoing myocardial ischemia, symptomatic hypotension, angina, or HF, if a rapid ventricular response does not respond promptly to pharmacological measure, immediate $\mathrm{CV}$ is recommended; in patient of early replace of $\mathrm{AF}$ after $\mathrm{CV}$, repeated current $\mathrm{CV}$ attempts may be made following. In patients with digitalis toxicity or hypokalemia, electrical $\mathrm{CV}$ is contraindicated $[14,15]$.

Pharmacological cardioversion can be used as followed: IV ibutilide is an effective drug available to covert AF, $1 \mathrm{mg}$ over 10 minutes while observing for QTc prolongation and ventricular proarrhythmia. Dose can be repeated after 10 minutes, but the risk of proarrhythmia increases [16-18]; or IV amiodarone loading dose 150 $\mathrm{mg}$ over 10 minutes + IV maintenance dose $0.5-1$ $\mathrm{mg} / \mathrm{min}$. It is an appropriate initial choice in patients with left ventricular hypertrophy (LVH), HF, or coronary heart disease (CHD), because it is associated with a low risk of proarrhythmia. Low-dose ( $\leq 200 \mathrm{mg}$ daily) is associated with fewer side effects than higher-dose regimens. Patients should be monitored regularly for thyroid, hepatic, and pulmonary toxicity.

\section{MAINTENANCE OF SINUS RHYTHM}

Before initiating antiarrhythmic drug (AAD) therapy, treatment of precipitating or reversible causes of $\mathrm{AF}$ is recommended. Pharmacological therapy can be useful in patients with AF to maintain SR and prevent tachycardia-induced cardiomyopathy [13]. Principles of AAD therapy are: The patients have troublesome symptoms related to paroxysmal $\mathrm{AF}$ or recurrent $\mathrm{AF}$ after $\mathrm{CV}$ who can tolerate $\mathrm{AAD}$, and have a good chance of remaining in SR; AAD choice is based on side effect profiles and the presence or absence of structural heart disease, HF, hypertension, should be individualized and must account for underlying renal and hepatic function. Outpatient initiation of AAD therapy is reasonable in patients with AF who have no associated heart disease and well tolerate AAD. Sotalol can be beneficial in outpatients in SR with mild or no heart disease, prone to paroxysmal AF, if 
the baseline uncorrected QT interval is less than $460 \mathrm{~ms}$, serum electrolytes are normal, and risk factors associated with Class III drug-related proarrhythmia are not present; ensure normal electrolyte status and appropriate anticoagulation prior to starting AAD therapy. Initiate therapy at low dose and titrate up as needed and after evaluating drug effects on ECG parameters. Initiate AV nodal blockade prior to use of AAD (e.g. flecainide) that do not provide substantial AV node blockade [6]. In patients with AF without structural or CHD, initiation of propafenone or flecainide can be beneficial on an outpatient basis in patients with paroxysmal AF who are in SR at the time of drug initiation [13] (Table 1).

In the recent guideline on the management of patients with AF $[6,13]$. Dronedarone recommends for the prevention of recurrent $\mathrm{AF}$, it is similar to amiodarone but lacks an iodine moiety, it has multiple electrophysiologic actions including sympatholytic effects and inhibition of the L-type calcium current, the inward sodium current, and multiple potassium current $[19,20]$, but it better tolerated [21,22]. Dronedarone can reduce the risk of cardiovascular hospitalization in patients with paroxysmal or persistent AF or atrial flutter (AFL), with a recent history of AF/AFL and associated cardiovascular risk factors (age $>70$, hypertension, diabetes, prior cerebrovascular accident, left atrium (LA) diameter $\geq 50 \mathrm{~mm}$ or LVEF $<40 \%$ ) who are in SR or who will be cardioverted [23], but recently, several cases of hepatocellular liver injury and hepatic failure in patients treated with dronedarone (marketed as Multaq), including two cases of acute hepatic failure requiring transplantation were reported [23].

\section{VENTRICULAR RATE CONTROL}

Most patients who used a rhythm control strategy also require medications for ventricular rate (VRs) control. Rate control during atrial flutter tends to be more difficult than

Table 1. The antiarrhythmic drug and only oral doses as follow.

\begin{tabular}{|c|c|c|}
\hline & Antiarrhythmic drug & Only oral dose \\
\hline \multirow{3}{*}{ Class I } & Flecainide & $50-150 \mathrm{mg}$ every 12 hours \\
\hline & Propafenone & 150 - $300 \mathrm{mg}$ every 8 hours \\
\hline & Propafenone SR & $225-425 \mathrm{mg}$ every 12 hours \\
\hline \multirow{4}{*}{ Class III } & Dronedarone & $200-400 \mathrm{mg}$ every 12 hours \\
\hline & Amiodarone & $\begin{array}{l}800 \mathrm{mg} / \text { day/1 week, then } 600 \\
\mathrm{mg} / \mathrm{day} / 1 \text { week, } \\
400 \mathrm{mg} / \text { day } / 4 \text { - } 6 \text { week, } 200 \mathrm{mg} / \text { day }\end{array}$ \\
\hline & Dofetilite & $\begin{array}{l}250-500 \text { mcg every } 12 \text { hours, based } \\
\text { on renal function, must be in the } \\
\text { hospital. }\end{array}$ \\
\hline & Sotalol & $\begin{array}{l}80-160 \mathrm{mg} \text { to maximum of } 320 \mathrm{mg} \text {, } \\
\text { every } 12 \text { hours, based on renal } \\
\text { function. }\end{array}$ \\
\hline
\end{tabular}

during AF. Control of the VRs during AF is important both at rest and exertion. Criteria vary with age but usually involve achieving VRs between 60 and $80 \mathrm{bpm}$ at rest and between 90 to $115 \mathrm{bpm}$ during moderate exercise or 6-minates walk test $[24,25]$. In some study, adequate control was defined as an average heart rate of up to $80 \mathrm{bpm}$ at rest and the maximum age-adjusted predicted exercise heart rate or a maximum heart rate of 110 bpm during a 6-minute walk test [26]. The potential benefits of strict (resting heart rate $<80 \mathrm{bpm}$, heart rate $<$ 110 bpm during moderate exercise) versus lenient (resting heart rate $<110 \mathrm{bpm}$ ) rate control were reported in the RACE II trial of 614 patients with permanent AF [27]. As lenient-rate control is generally more convenient, it may be adopted as a reasonable strategy in patients with permanent AF.

Several drugs can control the ventricular response in the different patients with AF: AV nodal blocking drugs that include Beta blockers: Esmolol is an ultra-shortacting beta- 1 receptors blocker, serum half-life of 9 minutes. Start with 60 -seconds loading dose of $0.5 \mathrm{mg} / \mathrm{kg}$ and follow with maintenance dose $60-200 \mathrm{mcg} / \mathrm{kg} / \mathrm{min}$. Metoprolol (Lopressor) is much more convenient to use, 2.5 - $5.0 \mathrm{mg}$ bolus over $2 \mathrm{~min}$ (up to 3 dose), oral dose is 25 - $100 \mathrm{mg}$ daily, or Atenolol 25 - $100 \mathrm{mg} / \mathrm{day}$, or Carvedilol 6.25 - $50 \mathrm{mg}$ every 12 hours, or Carvedilol SR 10 - $80 \mathrm{mg}$ daily orally. Calcium channel antagonist (nondihydropyridine) include Diltiazem- $0.25 \mathrm{mg} / \mathrm{kg}$ (average $20 \mathrm{mg}$ ) over $2 \mathrm{~min}$ ( $2^{\text {nd }}$ bolus can be given if $\mathrm{HR}>$ $100 \mathrm{bpm}$ ), 5 - $15 \mathrm{mg} /$ hours. Oral dose 120 - $480 \mathrm{mg}$ daily, and Verapamil $0.075-0.15 \mathrm{mg} / \mathrm{kg}$ over $2 \mathrm{~min}$. Oral dose: 120 - $480 \mathrm{mg}$ daily. Third drug is Digoxin $0.25 \mathrm{mg} \mathrm{q} 2$ hours, up to total $1.5 \mathrm{mg}$, maintenance dose: 0.125 $0.375 \mathrm{mg}$ daily orally.

\section{CATHETER ABLATION}

Catheter ablation to maintain SR in patients with AF is currently considered a second-line therapy in significantly symptomatic paroxysmal patients who have failed treatment with an AAD and have with normal size or mildly dilated atria, normal or mildly reduced ventricular function, and absence of severe pulmonary disease [28, 29]. Catheter ablation may be reasonable to treat symptomatic paroxysmal AF in patients with significant LA dilatation or with significant LV dysfunction [13]. Some younger patients with symptomatic AF who require SR, ablation may be preferred over years of drug therapy. Presence of a LA thrombus is a contraindication. Before ablation, we should evaluate AF etiology and underlying disease. Generally, reports describe success rates of $60 \%$ to $70 \%$ that approximately $10 \%$ to $40 \%$ of patients require a second ablation, and that $10 \%$ to $15 \%$ require continued antiarrhythmic therapy. 
In up to $90 \%$ of patients with AF, the "triggers" of arrhythmia arise from specific areas within the LA, near the openings of four pulmonary veins, when the openings of the veins isolated from the rest of the left atrium with radiofrequency catheter ablation by using a catheter to create just two or three strategically - placed linear scars, AF can often be reduced in frequency or even eliminated [30]. Despite these advances, the long-term efficacy of catheter ablation to prevent recurrent $\mathrm{AF}$ requires further study. Available data demonstrate 1 year or more of freedom from recurrent $\mathrm{AF}$ in most patients [31,32].

Complications are similar with other catheter-based intervention, such as infection, bleeding, hematoma, deep venous thrombosis, pneumothorax, and arterial damage. However, more serious complications are rare.

$\mathrm{AV}$ nodal ablation and permanent pacing is an option for patients with rapid ventricular rates despite maximum medical therapy to control the rate, otherwise AV nodal ablation should not be attempted. In the patients without underlying heart disease, survival among AF individuals after ablation of the AV node is similar to expected survival in the general population. Long-term survival is similar for patients with $\mathrm{AF}$ in whom received ablation or drug therapy. VRs controlled by ablation of the AV node and permanent pacing does not adversely affect longterm survival [33]. Catheter ablation of the AV conduction system and permanent pacemaker implantation are followed by improvement in the quality of life of severely symptomatic patients with $\mathrm{AF}$ that is refractory to medical treatment [34].

Biventricular pacing may overcome many of the adverse hemodynamic effects and a few cases of LV dysfunction that occurred with RV pacing alone. A target individual ectopic foci ablation within the PV has evolved to circumferential electrical isolation of the entire PV musculature. Catheter ablation of the cavotricuspid isthmus should be considered as first-line therapy for patients with typical atrial flutter. Radiofrequency ablation of the cavotricuspid isthmus was effective in eliminating typical atrial flutter without injury of antegrade fast AV node conduction. The atrial musculature in the cavo-tricuspid isthmus significantly contributed to the slow AV node conduction [35]. Completely non-fluoroscopic ablation guided by real-time magnetic resonance imaging (RTMRI) using a steerable and non-ferromagnetic catheter is a promising novel technology in interventional electrophysiology (EP) [36].

\section{ANTICOAGULANT COMBINED WITH ANTIPLATELET THERAPY}

Several studies have demonstrated that oral anticoagulation with warfarin is effective for prevention of thromboembolism in patients with AF [9,28,37]. Aspirin offers only modest protection against stroke for AF patients
[38-40]. Adjusted-dose oral anticoagulation is more efficacious than aspirin for prevention of stroke in AF patients [41]. Recent studies have assessed the thienopyridine antiplatelet agent clopidogrel with aspirin for stroke prevention in patients with AF [42-44]. The addition of clopidogrel to aspirin (ASA) to reduce the risk of major vascular events, including stroke, might be considered in patients with AF in whom oral anticoagulation with warfarin is considered unsuitable due to patient preference or the physician's assessment of the patient's ability to safely sustain anticoagulation [42]. The combined use of dual-antiplatelet therapy with both clopidogrel and ASA plus anticoagulation with warfarin (triple therapy) has been suggested as a strategy for treatment and prevention of complications of 2 or more coexisting conditions such as $\mathrm{AF}$, mechanical valve prosthesis, or the presence of a drug-eluting coronary stent [44].

All patients with AF regardless of whether a rhythm or rate control strategy recommend anticoagulation except those with lone AF or contraindications. Drug selection should be based upon the absolute risk of stroke, bleeding, the relative risk and benefit for a given patient (Tables 2 and 3 ).

Nonpharmacologic procedures to prevention of thromboembolism with Watchman device, 707 patients $\geq 18$ years of age (mean age $72 \mathrm{y}, 70 \%$ men) who had nonvalvular $\mathrm{AF}$ and CHADS2 risk score $\geq 1$ revealed that percutaneous closure of the left atrial appendage (LAA) was noninferior to warfarin therapy for efficacy but was associated with increased risk for the composite safety outcome, including serious pericardial effusion in $4.8 \%$ of patients [45]. FDA has not approved clinical use of the Watchman device pending the results of additional ongoing trials.

For all patients with $\mathrm{AF}$ for $>48$ hours, or when $\mathrm{AF}$

Table 2. The classification of risk factors.

\begin{tabular}{ccc}
\hline Weaker risk factors & Moderate risk factors & High risk factors \\
\hline Female & Age $\geq 75$ years & $\begin{array}{c}\text { Previous stroke, TIA, } \\
\text { or embolism }\end{array}$ \\
Age 65 to 74 years & Hypertension & Mitral stenosis \\
$\begin{array}{c}\text { Coronary artery } \\
\text { disease }\end{array}$ & Heart failure & Prosthetic cardiac valve \\
\hline
\end{tabular}

Table 3. Risk factors and recommended therapy.

\begin{tabular}{cc}
\hline Risk factors & Therapy \\
\hline None & Aspirin $81-325 \mathrm{mg}$ daily \\
1 moderate risk factor & $\begin{array}{c}\text { Aspirin } 81-325 \mathrm{mg} \text { daily, or } \\
\text { warfarin (INR2 }-3, \text { target 2.5) }\end{array}$ \\
$\begin{array}{c}\text { Any high risk factor or }>1 \\
\text { moderate risk factors }\end{array}$ & Warfarin (INR2 - 3, target 2.5) \\
\hline
\end{tabular}

INR (International Normalized Ratio) should be determined at least weekly when warfarin is initiated and monthly when INR is stable. 
duration is unknown, 3 weeks of therapeutic anticoagulation with an INR $\geq 2.0$ are required prior to $\mathrm{CV}$. It may take longer than 3 weeks. Anticoagulation (warfarin or heparin) must be continued for at least 4 weeks after CV regardless of the use of TEE before CV. Anticoagulation after 4 weeks dependent upon CHADS2 Risk Criteria Score: CHF +1 , hypertension history +1 , Age $\geq 75+1$, diabetes history +1 , stroke symptoms previously or TAI +2 . TEE can be used to assess the LA for thrombosis. Of the more than 700,000 strokes that occur in the US per year, approximately $15 \%$ are thought due to AF, while another $30 \%$ are of unknown cause and classified as cryptogenic [46]. Anticoagulation is highly effective in preventing embolic events in AF patients with a stroke risk reduction of almost $70 \%$ and is superior to other pharmacological approaches [47]. But anticoagulation might increase the risk of major bleeding by approximately $70 \%$ compared with aspirin, accounting for year [48]. Other disadvantages of anticoagulation therapy are its narrow therapeutic range, pharmacological and food interactions, and the need for frequent monitory and dose adjustment. These might be some of reasons why only $54 \%$ of all high-risk patients who are eligible for oral anticoagulation therapy actually receive warfarin [49].

In a recently randomized double-blind trial in 18201 AF patients from 39 countries who had at least one additional risk factor for stroke, studies compared a new oral anticoagulant - apixaban (5 mg twice daily) with warfarin (target INR 2.0 - 3.0), at a median follow-up of 1.8 years, revealed apixaban significantly reduced the relative risk of stroke or systemic embolism by 21 percent. The annual event rates were 1.27 percent for apixaban vs 1.60 percent for warfarin. Apixaban is a direct factor Xa inhibitor with predictable pharmacokinetics and a rapid onset and offset of action after oral administration [50]. In an another study for Rivaroxaban vs warfarin in nonvalvular AF, 14264 patients randomized received either rivaroxaban ( at a daily dose of $20 \mathrm{mg}$ ) or dose-adjusted warfarin (target INR 2.0 - 3.0), results revealed rivaroxaban was noninferior to warfarin for the prevent of stroke or systemic embolism [51].

\section{SURGICAL THERAPY}

The first surgical attempt to remove the LAA as a possible source for thromboembolic event was made by Madden in 1948 [52]. In 1991, Cox et al. introduced the "Maze" operation - several small incisions are made in the atria to interrupt AF reentry pathways [53]. In 2005, 197 patients underwent the Maze procedure were reported, the mean rate of disappearance of AF was $89 \%$ after 10 years follow up, but the Maze procedure is still not common used because of its complication at time. Complications of Maze operation include atrial dysfunc- tion, different types of atrial arrhythmias and no reduction for the risk of embolic events, the patients still have to continue with anticoagulation therapy.

The most common forms of ablative energy used in surgery for AF are high frequency ultra-sound (HIFU), radiofrequency energy and nitrous oxide-based cryotherapy ( -8 degree $C)$. All are capable of producing areas of localized complete myocardial ablation-electrical structural integrity [54].

\section{CONCLUSION}

$\mathrm{AF}$ is the most common clinically significant cardiac rhythm disorder, and a progressive disease with higher mortality consequences. The treatment with rhythm control or/and rate control and prevention of thromboembolism, it has been associated with improved survival, decreased hospitalization, improved cardiac function and quality of life. Nevertheless, the new treatments are still in the growing.

\section{REFERENCES}

[1] Naccarelli, G.V. (2007) Easing the burden and halting the progression of a persistent, yet highly treatable disorder. Research \& Review in Atrial Fibillation, 1, 2.

[2] Chen, L.Y. and Shen, W.K. (2007) Epidemiology of atrial fibrillation: A current perspective. Heart Rhythm, 4, 5156.

[3] Go, A.S., Hylek, E.M., Phillips, J.A., et al. (2001) Prevalence of diagnosed AF in adults: National implications for rhythm management and stroke prevent: The Anticoagulation and Risk Fraction in AF (ATRIA) Study. Journal of the American Medical Association, 285, 2370-2375. doi:10.1001/jama.285.18.2370

[4] Feinberg, W.M., Blackshear, J.L., Laupacis, A., et al. (1995) Prevalence age distribution and gender of patients with AF. Analysis and implacations. Archives of Internal Medicine, 155, 469-473. doi:10.1001/archinte.1995.00430050045005

[5] Miyasaka, Y., Barnes, M.E., Gersh, B.J., et al. (2006) Secular trends in incidence of AF in Olmsted County Minnesoda. 1980-2000, and implications on the projections for future prevalence. Circulation, 114, 119-125. doi:10.1161/CIRCULATIONAHA.105.595140

[6] Takshminarayan, K., Anderson, D.C., Qureshi, A.I., et al. (2008) Clinical epidemiology of AF and cerebrovascular events in the US. Neurologist, 14, 143-150. doi:10.1097/NRL.0b013e31815cffae

[7] Furberg, C.D., Psaty, B.M., Manolio, T.A., et al. (1994) Prevalence of AF in elderly subjects (the Cardiovascular Health Study). American Journal of Cardiology, 74, 236-241. doi:10.1016/0002-9149(94)90363-8

[8] Kannel, W.B., Wolf, P.A., Benjamin, E.L. and Levy, D. (1998) Prevalence, incidence, prognosis, and predisposing conditions for AF: Population-based estimates. American Journal of Cardiology, 82, 2N-9N. 
doi:10.1016/S0002-9149(98)00583-9

[9] Fuster, V., Rydén, L.E., David, S., Cannom, D.S., et al. (2006) ACC/AHA/ESC 2006 Guidelines for the management of patients with atrial fibrillation-Executive summary. Journal of the American College of Cardiology, 854-906. doi:10.1016/j.jacc.2006.07.009

[10] Allessie, M., Ausma, J. and Schotten, U. (2002) Electrical, contractile and structural remodeling during atrial fibrillation. Cardiovascular Research, 54, 230-246. doi:10.1016/S0008-6363(02)00258-4

[11] Kumagai, K., Nakashima, H., Urata, H., et al. (2003) Effects of angiotensin II type 1 receptor antagonist on electrical and structural remodeling in atrial fibrillation. Journal of the American College of Cardiology, 41, 21972204. doi:10.1016/S0735-1097(03)00464-9

[12] Dittrich, H.C., Erickson, J.S., Schneiderman, T., et al. (1989) Echocardiographic and clinical predictors for outcome of elective cardioversion of atrial fibrillation. American Journal of Cardiology, 63, 193-197. doi:10.1016/0002-9149(89)90284-1

[13] Wann, L.S., Curtis, A.B., January, C.T., et al. (2011) 2011 ACCF/AHA/HRS focused update on the management of patients with atrial fibrillation (updating the 2006 guideline): A report of the American college of cardiology foundation/American heart association task force on practice guidelines. Circulation, 123, 104-123. doi:10.1161/CIR.0b013e31820f14c0

[14] Fuster, V., Lars, E., Rydén, L.E., Cannom, D.S., et al. (2007) Management of patients with atrial fibrillation. ACC/AHA pocket guideline. The ACC/AHA/ESC guidelines for the management of patients with atrial fibrillation.

[15] Gallagher, M.M., Guo, X.-H., Poloniecki, J.D., et al. (2001) Initial energy setting, outcome and efficiency in direct current cardioversion of atrial fibrillation and flutter. Journal of the American College of Cardiology, 38, 1498-1450. doi:10.1016/S0735-1097(01)01540-6

[16] Mittal, S., Shcrvin, A., Stein, K.M., et al. (2000) Transthoracic cardioversion of atrial fibrillation: Comparison of rectilinear biphasic versus damped sine wave monophasic shocks. Circulation, 101, 1282-1287. doi:10.1161/01.CIR.101.11.1282

[17] Stambler, B.S., Wood, M.A. and Ellenbogen, K.A. (1996) Efficacy and safety of repeated intravenous doses of ibutilide for rapid conversion of atrial flutter or fibrillation. Ibutilide repeat dose study investigators. Circulation, 94, 1613-1621. doi:10.1161/01.CIR.94.7.1613

[18] Volgman, A.S., Carberry, P.A. and Stambler, B.S. (1998) Conversion efficacy and safety of intravenous ibutilide compared with intravenous procainamide in patients with atrial flutter or fibrillation. Journal of the American College of Cardiology, 31, 1414-1419. doi:10.1016/S0735-1097(98)00078-3

[19] Oral, H., Souza, J.J. and Michaud, G.F. (1999) Facilitating transthoracic cardioversion of atrial fibrillation with ibutilide pretreatment. The New England Journal of Medicine, 340, 1849-1854. doi:10.1056/NEJM199906173402401
[20] Patel, C., Yan, G.X. and Kowey, P.R. (2009) Dronedarone. Circulation, 120, 636-644. doi:10.1161/CIRCULATIONAHA.109.858027

[21] Piccini, J.P., Hasselblad, V., Peterson, E.D., et al. (2009) Comparative efficacy of dronedarone and amiodarone for the maintenance of sinus rhythm in patients with atrial fibrillation. Journal of the American College of Cardiology, 54, 1089-1095. doi:10.1016/j.jacc.2009.04.085

[22] Le Heuzey, J.Y., De Ferrari, G.M., Radzik, D., et al. (2010) A short-term, randomized, double-blind, parallelgroup study to evaluate the efficacy and safety of dronedarone versus amiodarone in patients with persistent atrial fibrillation: The DIONYSOS Study. Journal of Cardiovascular Electrophysiology, 21, 597-605. doi:10.1111/j.1540-8167.2010.01764.x

[23] Ellenbogen, K.A. (2011) Editor's choice: FDA warns of severe liver injury association with dronedarone. Cardio Source News, Top News.

[24] Atwood, J.E., Myers, J., Sandhu, S., et al. (1989) Optimal sampling interval to estimate heart rate at rest and during exercise in atrial fibrillation. American Journal of Cardiology, 63, 45-48.

[25] Tein, K.M., Borer, J.S., Hochreiter, C., et al. (1994) Variability of the ventricular response in atrial fibrillation and prognosis in chronic nonischemic mitral regurgitation. American Journal of Cardiology, 74, 906-911. doi:10.1016/0002-9149(94)90584-3

[26] Olshansky, B., Rosenfeld, L.E., Warner, A.L., et al. (2004) The Atrial Fibrillation Follow-Up Investigation of Rhythm Management (AFFIRM) study: Approaches to control rate in atrial fibrillation. Journal of the American College of Cardiology, 43, 1201-1208. doi:10.1016/j.jacc.2003.11.032

[27] Van Gelder, I.C., Groenveld, H.F., Crijns, H.J., et al. (2010) Lenient versus strict rate control in patients with atrial fibrillation. The New England Journal of Medicine, 362, 1363-1373. doi:10.1056/NEJMoa1001337

[28] Cappato, R., Calkins, H., Chen, S.A., et al. (2010) Updated worldwide survey on the methods, efficacy, and safety of catheter ablation for human atrial fibrillation. Circulation: Arrhythmia and Electrophysiology, 3, 32-38. doi:10.1161/CIRCEP.109.859116

[29] Bertaglia, E., Tondo, C., De Simone, A., et al. (2010) Does catheter ablation cure atrial fibrillation? Singleprocedure outcome of drug-refractory atrial fibrillation ablation: A 6-year multicentre experience. Europace, 12, 181-187. doi:10.1093/europace/eup349

[30] Fogoros, R.N. (2011) Ablating atrial fibrillation. About.com Guide.

[31] Karch, M.R., Zrenner, B., Deisenhofer, I., et al. (2005) Freedom from atrial tachyarrhythmias after catheter ablation of atrial fibrillation: A randomized comparison between 2 current ablation strategies. Circulation, 111, 2875-2880. doi:10.1161/CIRCULATIONAHA.104.491530

[32] Hindricks, G., Piorkowski, C., Tanner, H., et al. (2005) Perception of atrial fibrillation before and after radiofrequency catheter ablation: Relevance of asymptomatic ar- 
rhythmia recurrence. Circulation, 112, 307-313. doi:10.1161/CIRCULATIONAHA.104.518837

[33] Ozcan, C., Jahangir, A. and Friedman, P.A. (2001) Longterm survival after ablation of the atrioventricular node and implantation of a permanent pacemaker in patients with atrial fibrillation. The New England Journal of Medicine, 344, 1043-1051. doi:10.1056/NEJM200104053441403

[34] Kay, G.N., Ellenbogen, K.A., Giudici, M., et al. (1998) The ablate and pace trial: A prospective study of catheter ablation of the AV conduction system and permanent pacemaker implantation for treatment of atrial fibrillation. Journal of Interventional Cardiac Electrophysiology, 2, 121-135. doi:10.1023/A:1009795330454

[35] Tai, C.T., Tsai, C.F., Hsieh, M.H., et al. (2001) Effects of cavotricuspid isthmus ablation on atrioventricular node electrophysiology in patients with typical atrial flutter Circulation, 104, 1501-1505. doi:10.1161/hc3801.078813

[36] Hoffmann, B.A., Koops, A. and Rostock, T. (2010) Interactive real-time mapping and catheter ablation of the cavotricuspid isthmus guided by magnetic resonance imaging in a porcine model. European Heart Journal, 31, 450456. doi:10.1093/eurheartj/ehp460

[37] The Boston Area Anticoagulation Trial for Atrial Fibrillation Investigators (1996) The effect of low-dose warfarin on the risk of stroke in patients Prevention in Atrial Fibrillation Investigators. Adjusted-dose warfarin versus lowintensity, fixed-dose warfarin plus aspirin for high-risk patients with atrial fibrillation: Stroke Prevention in Atrial Fibrilation III randomised clinical trial. Lancet, 348, 633638. doi:10.1016/S0140-6736(96)03487-3

[38] Posada, I.S. and Barriales, V. (1999) Alternate-day dosing of aspirin in atrial fibrillation. LASAF Pilot Study Group. American Heart Journal, 138, 137-143. doi:10.1016/S0002-8703(99)70259-0

[39] Stroke Prevention in Atrial Fibrillation Investigators (1994) Warfarin versus aspirin for prevention of thromboembolism in atrial fibrillation: Stroke Prevention in Atrial Fibrillation II Study. Lancet, 343, 687-691.

[40] Hellemons, B.S., Langenberg, M., Lodder, J., et al. (1999) Primary prevention of arterial thromboembolism in nonrheumatic atrial fibrillation in primary care: Randomised controlled trial comparing two intensities of coumarin with aspirin. British Medical Journal, 319, 958-964. doi: $10.1136 / \mathrm{bmj} .319 .7215 .958$

[41] Hart, R.G., Benavente, O., McBride, R. and Pearce, L.A. (1999) Antithrombotic therapy to prevent stroke in patients with atrial fibrillation: A meta-analysis. Annals of Internal Medicine, 131, 492-501.

[42] Connolly, S., Pogue, J., Hart, R., et al. (2006) Clopidogrel plus aspirin versus oral anticoagulation for atrial fibrilla- tion in the Atrial fibrillation Clopidogrel Trial with Ibesartan for prevention of Vascular Events (ACTIVE W): A randomised controlled trial with Ibesartan for prevention of Vascular Events (ACTIVE W): A randomised controlled trial. Lancet, 367, 1903-1912.

doi:10.1016/S0140-6736(06)68845-4

[43] Connolly, S.J., Pogue, J., Hart, R.G., et al. (2009) Effect of clopidogrel added to aspirin in patients with atrial fibrillation. The New England Journal of Medicine, 360, 2066-2078. doi:10.1056/NEJMoa0901301

[44] Holmes, D.R. Jr., Kereiakes, D.J., et al. (2009) Combining antiplatelet and anticoagulant therapies. Journal of the American College of Cardiology, 54, 95-109. doi:10.1016/j.jacc.2009.03.044

[45] Holmes, D.R., Reddy, V.Y., Turi, Z.G., et al. (2009) Percutaneous closure of the left atrial appendage versus warfarin therapy for prevention of stroke in patients with atrial fibrillation: A randomised non-inferiority trial. Lancet, 374, 534-542. doi:10.1016/S0140-6736(09)61343-X

[46] Gulcher, J. (2008) From bad to worse. The conference connection. Highlights of the AHA Scientific Sessions, New Orleans, 1, 4-5.

[47] Hart, R.G., Halperin, J.L., Peace, L.A., et al. (2003) Lessons from the stroke prevention in AF trials. Annals of Internal Medicine, 138, 831-838.

[48] Levine, M.N., Raskob, G., Landefeld, S., et al. (2001) Hemorrhygic complication of anticoagulant treatment Chest, 119, 108s-121s. doi:10.1378/chest.119.1_suppl.108S

[49] Waldo, A.L., Becker, R.C., Tapson, V.F., et al. (2005) Hospitalized patients with AF and high risk of stroke are not being provided with adequate anticoagulation agents. Journal of the American College of Cardiology, 46, 17291736. doi:10.1016/j.jacc.2005.06.077

[50] ARISTOTLE trial (2011) New oral anticoagulant apixaban versus warfarin in atrial fibrillation. The New England Journal of Medicine, 365, 765-775.

[51] Patel, M.R., Mahaffey, K.W., Garg, J., et al. (2011) Rivaroxaban versus warfarin in nonvalvular atrial fibrillation. The New England Journal of Medicine, 365, 883891. doi:10.1056/NEJMoa1009638

[52] Madden, J. (1948) Resection of left atrial appendix. Journal of the American Medical Association, 140, 769772. doi:10.1001/jama.1949.02900440011003

[53] Cox, J.L., et al. (1991) Maze operation in patients with atrial fibrillation. Journal of Thoracic and Cardiovascular Surgery, 101, 406-426.

[54] Lawric, G.M. (2008) Surgery for atrial fibrillation. JMDHVC, 4, 8-11. 